\title{
Estrategias de control no lineal para la regulación de corriente y estabilización de voltaje DC para un aerogenerador operando en una microred \\ Non-linear control strategies for current regulation and DC voltage stabilization for a wind turbine operating in a microgrid
}

\author{
Pablo Proaño (iD ${ }^{1 *}$, Oscar Camacho (iD) ${ }^{2}$, Marcelo Pozo (iD ${ }^{2}$ \\ ${ }^{1}$ Escuela de Formación de Tecnólogos, Escuela Politécnica Nacional, Quito, Ecuador, 170525 \\ ${ }^{2}$ Departamento de Automatización y Control Industrial, Escuela Politécnica Nacional, Quito, Ecuador, 170525; \\ oscar.camacho@epn.edu.ec; marcelo.pozo@epn.edu.ec
}

* Correspondencia: pablo.proano@epn.edu.ec

Recibido 18 noviembre 2019; Aceptado 05 febrero 2020; Publicado 01 junio 2020

Resumen: La generación de energía eléctrica a partir de energías renovables, en especial eólica, se encuentra ocupando cada vez más una mayor participación en el mercado energético a nivel mundial. Debido a la alta dinámica del viento, se presentan variaciones en la magnitud de voltaje y frecuencia del sistema que dificulta integrar estos generadores al sistema eléctrico. Varios trabajos proponen diferentes estrategias de control implementadas en sistemas electrónicos de potencia para solventar este problema. Estos sistemas primero rectifican el voltaje variable del generador creando un acople de corriente directa (DC) y luego convierten el resultado en voltaje con magnitud y frecuencia constante. Los controladores más usados son de tipo proporcional integral derivativo (PID), los cuales presentan un buen desempeño en zonas de operación lineal. Sin embargo, existen propuestas no lineales que presentan amplias ventajas frente a los PIDs, sobre todo en sistemas con dinámicas rápidas y no lineales. En el presente trabajo se implementan controladores PIDs lineales y se proponen dos estrategias de control no lineales, una con una estructura PID y otra con control de modo deslizante (SMC), para regular el suministro de corriente y voltaje en aerogeneradores conectados a un sistema electrónico de potencia. En los controladores propuestos se implementó una ganancia no lineal que se calcula en función del error del sistema y se comparó su desempeño con el de los controladores PIDs lineales. Los resultados mostraron mejoras notables en la velocidad de estabilización del sistema y reducción de oscilaciones ante variaciones repentinas de referencia y perturbaciones externas. Para probar el desempeño de los controladores se simuló una micro red enlazada a una barra infinita en Simulink.

Palabras clave: Control de modo deslizante, control no lineal, sistemas eléctricos de potencia, turbina eólica.

Abstract: $\quad$ The generation of electrical energy from renewable energies, especially wind, is taking greater participation in the energy market worldwide. Due to the high wind dynamics, there are variations in the system's frequency and voltage, making it challenging to integrate these generators into the electrical system. Several works propose different control strategies implemented in electronic power systems to solve this problem. These systems first rectify the generator's variable voltage, creating a direct current (DC) link and then transforming it into voltage with constant magnitude and frequency. The most common controllers are PID (proportional integral derivative), which have a good performance in linear operation areas. However, there are non-linear proposals with broad advantages over PIDs, especially in systems with fast and non-linear dynamics. In this study, linear PID controllers are implemented. Two non-linear control strategies are proposed, one with a PID structure and the other with sliding mode control (SMC), to regulate the current and voltage supply in wind turbines connected to electronic system power. A non-linear gain was implemented in the proposed controllers, which is calculated based on the system error; their performance was compared with linear PID controllers. The results showed notable improvements in the system's stabilization speed and reduction of oscillations in the face of sudden reference variations and external disturbances. A micro-network linked to an infinite bar was simulated in Simulink to test the controllers' performance.

Keywords: $\quad$ Electric power systems, non-linear control, sliding mode control, wind turbine. 


\section{Unach}

\section{$1 \quad$ Introducción}

En la actualidad, la generación de energía eléctrica a partir de fuentes de energía renovable ocupa cada vez una mayor proporción a nivel mundial entre las fuentes de generación en sistemas eléctricos de potencia (Pöschke, Fortmann, \& Schulte, 2017). Una de las fuentes más comunes de generación de energía renovable es la eólica.

Para que un aerogenerador entregue energía a un sistema eléctrico con frecuencia y voltaje constante son necesarias varias etapas intermedias entre el generador y la red. Estas etapas incluyen comúnmente un rectificador de voltaje, un enlace de corriente directa (DC) y un inversor de potencia, cuyo control generalmente es de tipo proporcional integral derivativo (PID). Abbas, Abdulsada, \& Abusief (2011) y Qi \& Meng (2012) prueban y recomiendan el uso de estos controladores PID para el control de generadores eólicos.

Los controladores PID se encuentran en el 95\% de los procesos de control; sin embargo, poseen algunas desventajas frente a otras técnicas, una de ellas se estudia en Fernández (2011) y es la sintonización de los parámetros de regulación, que si no se calculan adecuadamente provocarán sobre impulsos en la respuesta y errores u oscilaciones en estado estable. Otra desventaja de los controladores PID estudiada por Boukhezzar \& Siguerdidjane (2010) es que son aplicables únicamente a sistemas o plantas lineales, esto dificulta su implementación, ya que una gran porción de procesos, sensores, transductores y elementos finales de control presentan características no lineales o son propensos a perder su linealidad debido a factores externos como el envejecimiento, temperatura, saturación magnética, entre otros. Por ejemplo, una turbina eólica se la puede modelar de manera lineal, pero en ciertos puntos de operación puede cambiar su linealidad.

Además, Boukhezzar \& Siguerdidjane (2010) concluyen que acciones lentas o con ganancias muy fuertes de regulación implementadas en los controladores que se encuentran en sistemas eólicos pueden provocar desestabilización tanto en la máquina como en el sistema eléctrico, esto debido a la rápida respuesta característica de un sistema eléctrico de potencia.

En la última década se han propuesto varias estructuras de control no lineal que superen las limitaciones de los controladores PID. Algunas propuestas se han orientado a plantear estructuras con ganancias variables, ganancias no lineales o métodos de auto sintonización que permiten a los controladores mejorar su adaptabilidad y robustez. Pöschke et al. (2017) proponen y prueban este tipo de estrategias para el desarrollo de controladores PID-adaptativos implementados en aerogeneradores.

Su, Sun, \& Duan (2005) proponen un controlador de tipo PID que tiene una ganancia no lineal, la cual es función del error y hace que el controlador se comporte como un PID lineal si el error actual es menor que el error máximo y como un controlador ON-OFF cuando el error actual supera al error máximo. Como resultado se obtuvo una notable mejora de rendimiento y alta robustez con respecto al ruido frente a un sistema de control PID con ganancia fija.

Así mismo, Lei \& Zhou (2018) proponen una estructura de PID basada en funciones no lineales de Ham, en donde la ganancia del controlador PID se calcula usando constantes lineales y funciones no lineales, donde los resultados muestran que es capaz de alcanzar las referencias con menor variación y con menor sobre impulsos a diferencia de su contraparte lineal.

Otra técnica muy usada en los últimos años en sistemas lineales y no lineales son las técnicas de control por modos deslizantes (SMC). Capito, Proaño, Camacho, Rosales, \& Scaglia (2016) compararon controladores deslizantes con controladores PID y se concluye que los primeros son más rápidos y robustos ante perturbaciones y errores de modelado que los PID clásicos.

Camacho \& Smith (2000) proponen un SMC para procesos químicos diseñado desde una superficie deslizante tipo PID y un modelo reducido de primer orden de la planta con retardo de tiempo (FOPDT) con nuevos parámetros de ajuste definidos como funciones de los parámetros característicos del FOPDT, en donde en base a los ejemplos presentados por los autores se evidencia que el rendimiento del controlador es satisfactorio a pesar de la no linealidad del sistema y una amplia gama de condiciones de operación.

Así mismo, Liu et al. (2018) proponen técnicas SMC para el control de turbinas eólicas de baja velocidad con una ley de alcance exponencial. Los autores usan modelos no lineales para probar el rendimiento del controlador y concluyen que los controladores SMC presentan un mejor desempeño que los controladores tradicionales con características lineales; además, Bianconi et al. (2013) diseñaron un SMC para el control de corriente en sistemas de energía renovable y hacen énfasis en que dada la rápida naturaleza de los sistemas eléctricos, los controladores de corriente requieren técnicas más rápidas que los controladores convencionales PID.

La contribución de este trabajo es proponer dos estrategias de control no lineal para regular la corriente suministrada a la red y el voltaje del enlace 


\section{Unach}

DC de un aerogenerador conectado a la red eléctrica mediante un sistema electrónico de potencia.

Para los controladores de corriente que modulan las señales de control del inversor de acople a la red eléctrica y que necesitan tener técnicas de control más rápidas que los de voltaje, se propone comparar un controlador PID clásico con un SMC que use una variación de la estructura propuesta por Camacho \& Smith (2000) y a la que se le implementará una ganancia no lineal que sea función del error del sistema.

Para el controlador de voltaje que están en cascada al controlador de corriente y que modulan el voltaje del acople DC del sistema se propone comparar un PID clásico con un PID no lineal que tenga una ganancia variable en función del error del sistema.

La implementación y comparación de los controladores se hará por medio de la librería computacional "Simscape Electrical" de Simulink en Matlab®. Para la comparación del desempeño de los controladores propuestos se usará el índice de error absoluto integral (IAE) propuesto por RodríguezMariano et al. (2015); este índice es útil cuando se quiere comparar la velocidad de los controladores en llegar a un estado estable.

\section{Metodología}

\subsection{Sistema de Pruebas}

Se implementó un sistema de pruebas haciendo uso de la librería "Simscape Electrical" de Simulink en Matlab®. En el sistema de pruebas se pretende evaluar el desempeño de controladores tipo PID lineal y dos propuestas no lineales, la una con una estructura PID y la otra con una estructura SMC. Para poder evaluar el desempeño de los contralores cuando el sistema entrega potencia y simular perturbaciones se conectó una planta de generación a través de una línea de transmisión de $30(\mathrm{~km})$, y transformadores elevadores reductores a un bus infinito, tal como se muestra en la fFigura 1.

Para la simulación de la generación se implementó un aerogenerador de inducción de polos salientes, un sistema de rectificación, un conversor elevador DC/DC, un inversor, filtro e inductancia de acople a la red, tal como se muestra en la figura 2.

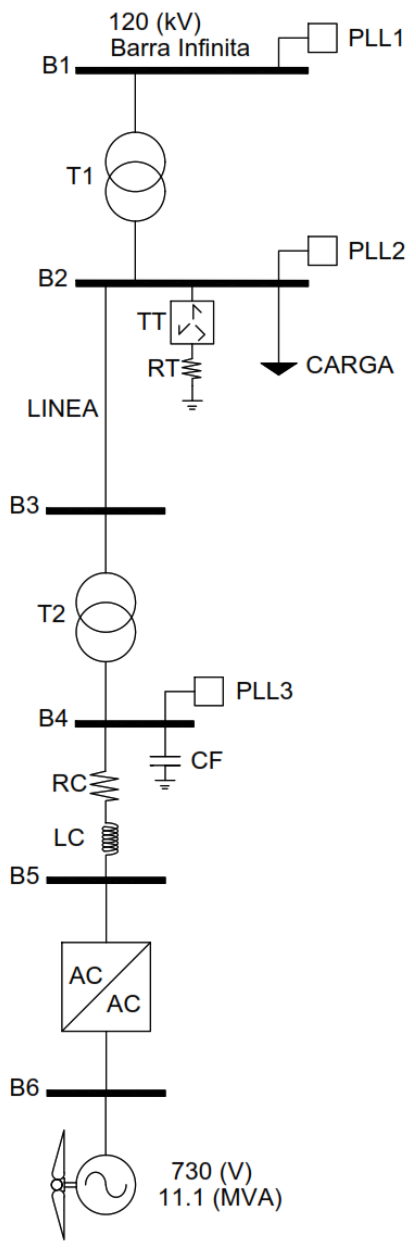

Figura 1: Sistema para la Simulación del Aerogenerador.

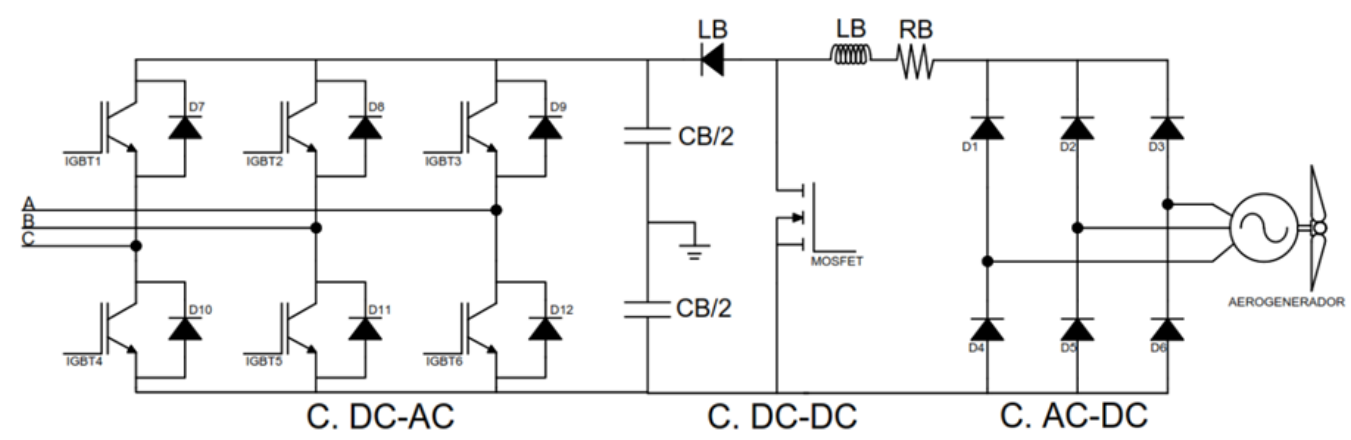

Figura 2: Sistema Electrónico de Potencia. 


\section{Unach}

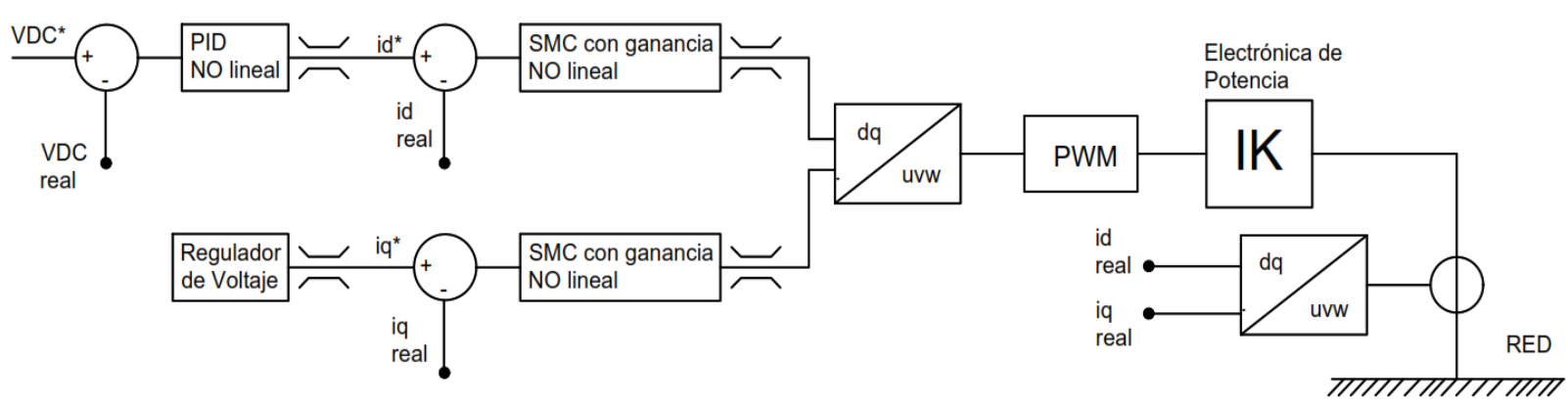

Figura 3: Estructura de control en cascada usada para el control de corriente y voltaje.

Para controlar la corriente y el voltaje se usó una estructura en cascada, en donde en el lazo interno se controla la corriente en eje directo y de cuadratura del inversor de voltaje, y en un lazo externo se controla el voltaje del bus DC regulado por el conversor elevador DC/DC.

En la figura 3 se puede apreciar el esquema en cascada del control, en donde la salida del controlador de voltaje DC se convierte en referencia para el control de corriente en eje directo, además la referencia de corriente en eje de cuadratura viene de una librería para la regulación del voltaje terminal del generador en alterna.

Dado que se usa un generador de polos salientes, el circuito equivalente en eje de cuadratura y directo son diferentes; por ende, se usaron controladores de igual estructura, pero con diferentes constantes de sintonización para ambas corrientes.

Una vez implementada la estructura de control, se implementaron PIDs lineales para la regulación de la corriente y voltaje. Se ingresaron referencias al sistema y se sometió a perturbaciones de voltaje en la barra de conexión al bus infinito, finalmente se almacenó los resultados para comparar los resultados obtenidos con los de los controladores no lineales propuestos.

\subsection{Control no lineal de corriente por modo deslizante}

La corriente es una variable eléctrica que es rápida y posee una alta dinámica, entonces para poder regularla se necesita un controlador que sea rápido y robusto.

Por lo anterior expuesto se escogió una estructura SMC para el control de corriente, además el modelado de esta en eje directo y de cuadratura resulta sumamente dificultoso debido al número de componentes no lineales que posee el sistema de generación, por esta razón se la aproximó a un FOPDT y se seleccionó un controlador que tenga robustez ante errores de modelado.

\subsubsection{Estructura del controlador SMC}

La estructura SMC usada en el presente trabajo es la propuesta por Camacho \& Smith (2000), la cual usa una superficie deslizante tipo PID que se muestra en (1).

$$
\mathrm{S}(\mathrm{t})=\frac{\mathrm{de}(\mathrm{t})}{\mathrm{dt}}+\lambda_{1} \mathrm{e}(\mathrm{t})+\lambda_{0} \int_{0}^{\mathrm{t}} \mathrm{e}(\mathrm{t})
$$

donde $\lambda_{0}$ y $\lambda_{1}$ son constantes y e(t) es el error del sistema.

Un controlador SMC posee una componente continua (lineal) y una discontinua (no lineal).

La parte discontinua del controlador generalmente se implementa mediante una función signo, sin embargo, esto provoca ruido en la acción de control de una amplitud que puede averiar los elementos finales de control, en (Camacho \& Smith, 2000) se propone usar una función de tipo sigmoide para mitigar este efecto, la cual se muestra en la ecuación (2).

$$
\mathrm{U}_{\mathrm{D}}(\mathrm{t})=\mathrm{K}_{\mathrm{D}} \frac{\mathrm{S}(\mathrm{t})}{|\mathrm{S}(\mathrm{t})|+\delta}
$$

donde $\mathrm{K}_{\mathrm{D}} \mathrm{y} \delta$ son contantes.

La parte continua del control se encarga de mantener al sistema montado sobre la referencia y se obtiene derivando la superficie de deslizamiento, igualándola a cero y remplazando el resultado en el modelo aproximado FOPDT del sistema, obteniéndose la ley de control que muestra en la ecuación (3).

$$
\mathrm{U}_{\mathrm{C}}(\mathrm{t})=\frac{\mathrm{X}(\mathrm{t})}{\mathrm{k}}+\frac{\mathrm{t}_{0} \tau}{\mathrm{k}} \lambda_{0}(\mathrm{R}(\mathrm{t})-\mathrm{X}(\mathrm{t}))
$$




\section{Unach}

donde $\mathrm{t}_{0}, \tau$ y $\mathrm{k}$ son los parámetros característicos del FOPTD.

La ecuación final del controlador SMC usado para los controles de corriente se muestra en la ecuación (4).

$$
\begin{gathered}
U(t)=\frac{\mathrm{X}(\mathrm{t})}{\mathrm{k}}+\frac{\mathrm{t}_{0} \tau}{\mathrm{k}} \lambda_{0}(\mathrm{R}(\mathrm{t})-\mathrm{X}(\mathrm{t}))+ \\
\mathrm{K}_{\mathrm{D}} \frac{\mathrm{S}(\mathrm{t})}{|\mathrm{S}(\mathrm{t})|+\delta}
\end{gathered}
$$

Camacho \& Smith (2000) presentan la justificación y el proceso completo para la obtención de las leyes de control expresadas en las ecuaciones (2), (3) y (4); en el presente trabajo solo se muestra de forma muy resumida la obtención de las mismas.

\subsubsection{Modificación propuesta a la estructura SMC}

La modificación propuesta a la configuración anterior es hacer que la ganancia de la parte discontinua sea función del error y por ende no lineal, tal como se ve en la ecuación (5). De esta manera se pretende que la acción discontinua tenga una influencia muy grande en el control cuando el error es alto y una acción muy pequeña cuando el error tiende a cero.

$$
\mathrm{K}_{\mathrm{D}}^{\prime}=\mathrm{K}_{\mathrm{D}}(1+|\mathrm{e}(\mathrm{t})|)
$$

Dado que la acción discontinua se encarga de llevar al sistema a la referencia a la mayor velocidad posible, puede provocar oscilaciones en el sistema, con la ganancia no lineal se pretende que el sistema sea rápido frente a cambios bruscos de referencia sin que presente oscilaciones por la acción de control.

Quedando la parte discontinua como se muestra en la ecuación (6), donde cabe resaltar que cuando el error tienda a cero la parte discontinua del controlador queda como la propuesta por Camacho \& Smith (2000).

$$
\mathrm{U}_{\mathrm{D}}(\mathrm{t})=\mathrm{K}_{\mathrm{D}}(1+|\mathrm{e}(\mathrm{t})|) \frac{\mathrm{S}(\mathrm{t})}{|\mathrm{S}(\mathrm{t})|+\delta}
$$

La sintonización de las constantes de los controladores se realizó mediante algoritmos genéticos, en donde las variables de decisión del sistema fueron las constantes del controlador y como función objetivo se usó el error del sistema al seguir la referencia. Se implementaron dos etapas; en la primera se sintonizó los controladores usando el error absoluto como función objetivo para corregir el error en estado estable y en la segunda etapa se sintonizó las constantes usando el error cuadrático como función objetivo para disminuir los sobre impulsos.
Finalmente se realizó una calibración fina de las constantes de los controladores.

\subsection{Control no lineal de voltaje de tipo PID}

El voltaje es una variable eléctrica más lenta que la corriente, por ende, no necesita una estructura rápida y relativamente compleja como es el SMC. Se implementó la ganancia no lineal descrita en la ecuación (5) en una estructura relativamente sencilla de tipo PID que le permita al sistema aumentar su tiempo de respuesta sin aumentar las oscilaciones.

\subsubsection{Estructura del controlador PID}

Para el estudio se empezó implementando una estructura PID clásica, la cual se puede ver ecuación (7):

$$
\mathrm{U}_{\mathrm{PI}}(\mathrm{t})=\mathrm{k}_{\mathrm{p}} \mathrm{e}(\mathrm{t})+\mathrm{k}_{\mathrm{i}} \int_{\mathrm{o}}^{\mathrm{t}} \mathrm{e}(\mathrm{t})+\mathrm{k}_{d} \frac{\mathrm{de}(\mathrm{t})}{\mathrm{dt}}
$$

donde $\mathrm{k}_{\mathrm{p}}, \mathrm{k}_{\mathrm{i}} \mathrm{y} \mathrm{k}_{\mathrm{d}}$ son constantes del controlador.

\subsubsection{Modificación propuesta a la estructura original PID}

Para el controlador con estructura PID no lineal se multiplicó a todo el controlador por la ganancia no lineal mostrada en la ecuación (5). Esto para que, igual que en la sección anterior, el sistema tenga acciones de control fuertes cuando el error del sistema es alto y acciones de control leves cual el sistema ya haya alcanzado la referencia. De esta manera, la ley de control queda como se observa en (8).

$$
\begin{gathered}
\mathrm{U}_{\mathrm{PI}}(\mathrm{t})=\mathrm{K}_{\mathrm{D}}^{\prime}\left(\mathrm{k}_{\mathrm{p}} \mathrm{e}(\mathrm{t})+\mathrm{k}_{\mathrm{i}} \int_{\mathrm{o}}^{\mathrm{t}} \mathrm{e}(\mathrm{t})\right. \\
\left.+\mathrm{k}_{d} \frac{\mathrm{de}(\mathrm{t})}{\mathrm{dt}}\right)
\end{gathered}
$$

La sintonización de las constantes se hizo siguiendo un procedimiento similar implementado para SMC, donde primero se sintonizó para error en estado estable, luego para sobre impulsos y finalmente se hizo una calibración fina

\subsection{Perturbaciones ingresadas al sistema}

Un aspecto muy importante en el diseño de controladores es evaluar si la configuración de control es resistente ante perturbaciones externas y que tan estable es el sistema ante dichas perturbaciones.

En el presente trabajo se va a usar como perturbación externa al sistema de generación una caída de voltaje 


\section{Unach}

en los terminales de la barra infinita, la cual será mitigada por el controlador del bus DC que se encuentra en cascada con el controlador de corriente.

\section{$3 \quad$ Resultados y Discusión}

En el presente estudio se optó por no trabajar con las magnitudes reales de las variables a controlar, sino que se calculó los valores por unidad de estas, esto para que el proceso de sintonización sea el mismo para corriente y voltaje.

En primer lugar, se probó el desempeño del controlador SMC con la ganancia no lineal implementada en la componente discontinua del mismo. Para eso se ingresó una señal de entrada tipo pulso de magnitud $0.5 \mathrm{pu}$ en el tiempo $0.15 \mathrm{~s}$ con una duración de $0.15 \mathrm{~s}$ y se comparó la respuesta con la del controlador PID lineal previamente implementado. La misma señal de entrada fue usada para la corriente en eje directo y eje de cuadratura.

En la figura 4 se muestra la respuesta de la corriente en eje directo y en la figura 6 se muestra un acercamiento a dicha respuesta. En la comparación se puede apreciar que la propuesta SMC con la ganancia no lineal (negro) tienen un sobre impulso más pequeño, se estabiliza más rápido y con menos oscilaciones que el PID clásico (rojo). Para evaluar la mejora en el desempeño del controlador SMC en contra del PID lineal se procedió a calcular el error acumulado integral (IAE) para cada esquema de control, en la tTabla 1 se muestran estos índices y la mejora obtenida de aproximadamente 5\%.

Tabla 1: Desempeño de la propuesta SMC y del controlador PID lineal de la corriente en eje directo.

\begin{tabular}{cc} 
Control Implementado & IAE \\
\hline PID lineal & 133.3144 \\
SMC con ganancia no lineal & 119.1582 \\
Mejora obtenida & $5.16 \%$ \\
\hline
\end{tabular}

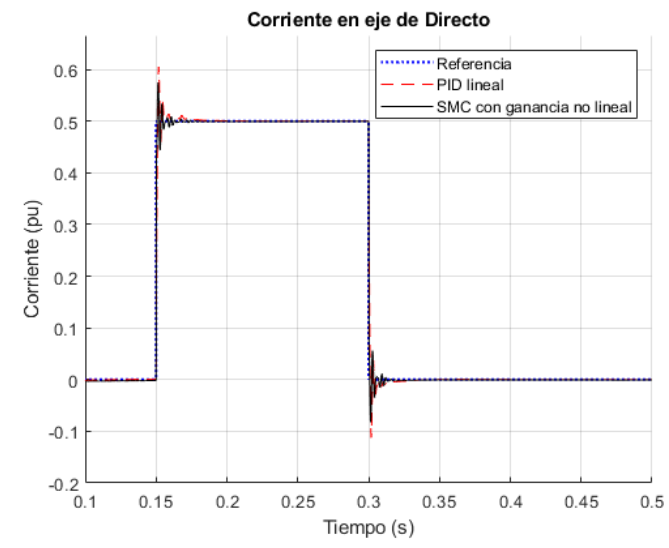

Figura 4: Respuesta de corriente en eje directo ante un pulso en la entrada.
En la figura 5 se muestra la respuesta de la corriente en eje de cuadratura y en la fFigura 7 se muestra un acercamiento a esta respuesta. De igual manera que en eje directo, la respuesta de la propuesta con ganancia no lineal tiene un sobre impulso más pequeño, se estabiliza más rápido y con menos oscilaciones que el PID clásico.

Del mismo modo que en el eje directo, se calculó el error acumulado integral para el control SMC y el PID lineal. En la tabla 2 se muestran los índices calculados y la mejora obtenida de aproximadamente $33 \%$.

Tabla 2: Desempeño de la propuesta SMC y del controlador PID lineal de la corriente en eje de cuadratura.

\begin{tabular}{cc} 
Control Implementado & IAE \\
\hline PID lineal & 46.0199 \\
SMC con ganancia no lineal & 30.3920 \\
Mejora obtenida & $32.81 \%$ \\
\hline
\end{tabular}

Luego, para evaluar el desempeño del controlador PID no lineal que tiene la ganancia como función del error y que multiplica a la acción de control original, se ingresó una señal de entrada de tipo escalón de $1 p u$ de magnitud en el tiempo $0 \mathrm{~s}$ y se procedió a evaluar la respuesta del sistema en contra de la respuesta de un controlador PID lineal previamente implementado.

En la figura 8 se muestra la respuesta del voltaje DC del bus de enlace entre el inversor y el rectificador, donde se puede notar una gran mejora en el desempeño del PID no lineal (negro) en contra del PID lineal (rojo).

La mejora no solo se debe a la dinámica rápida del controlador no lineal, sino también a que el controlador de voltaje se encuentra en cascada con los de corriente y en estos también se encuentran implementadas estrategias de control con la ganancia no lineal (calculada en función del error).

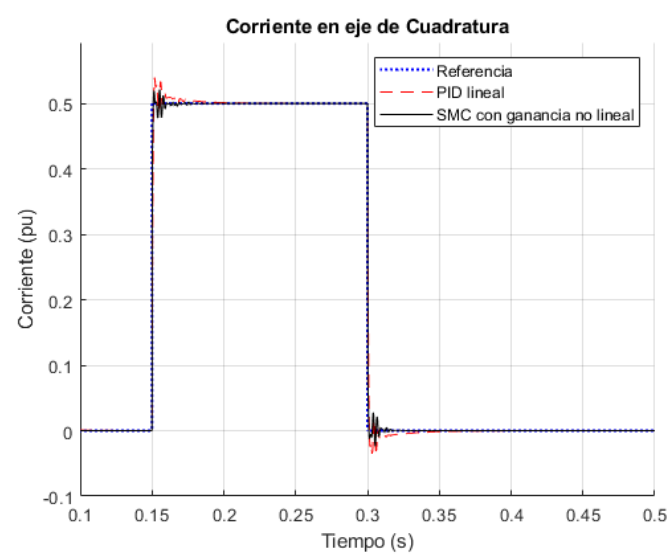

Figura 5: Respuesta de corriente en eje de cuadratura ante un pulso en la entrada. 


\section{Unach}

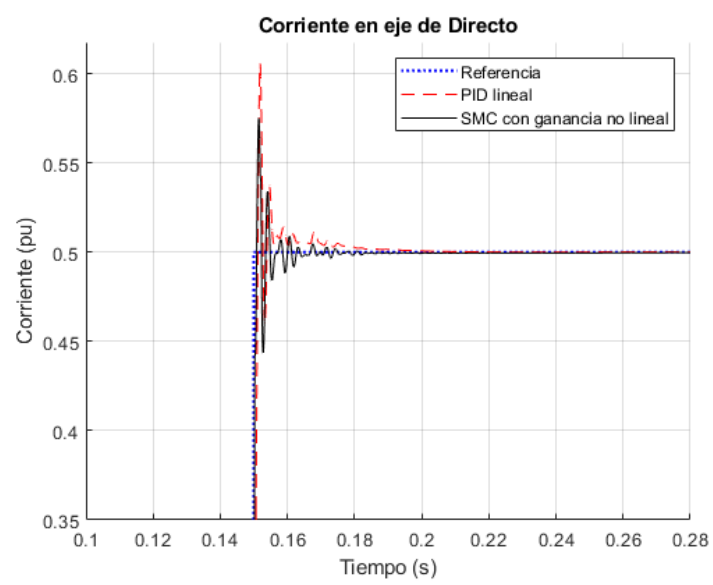

Figura 6: Acercamiento a la respuesta de corriente en eje directo ante un pulso en la entrada.

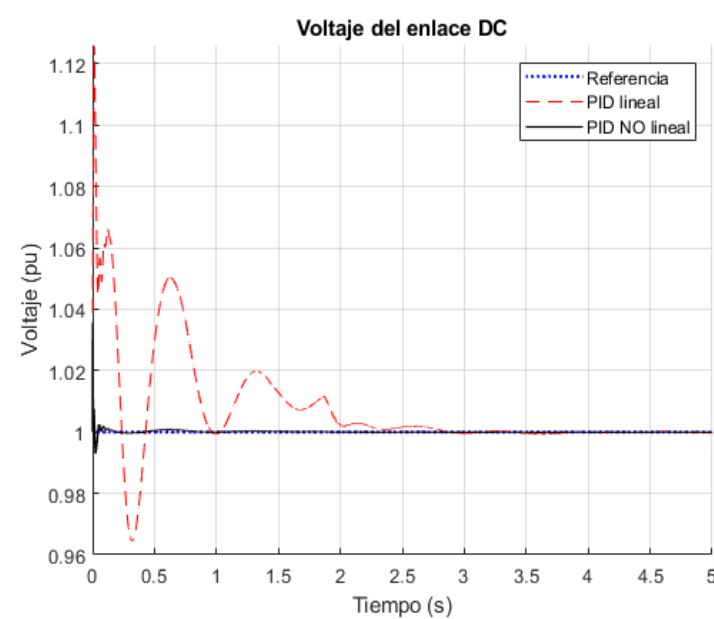

Figura 8: Respuesta del Voltaje DC ante una entrada de tipo escalón.

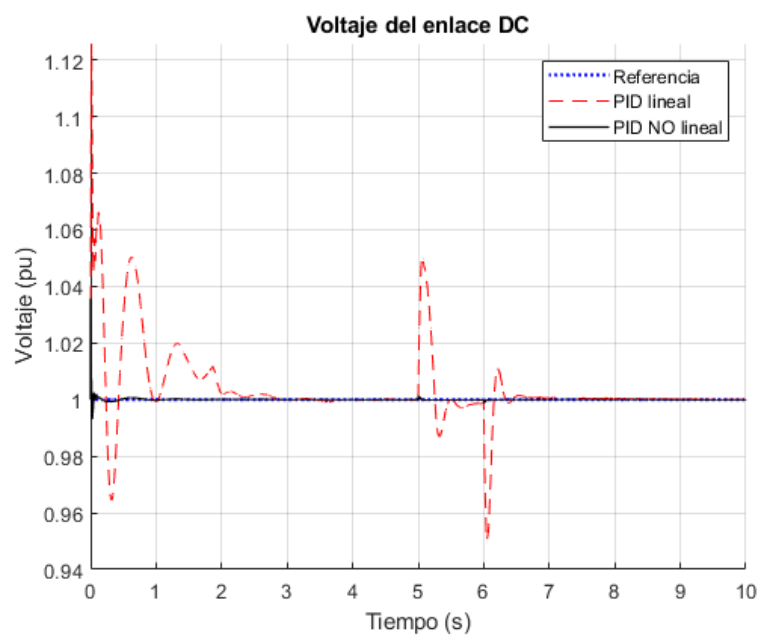

Figura 9: Respuesta del sistema ante una perturbación.

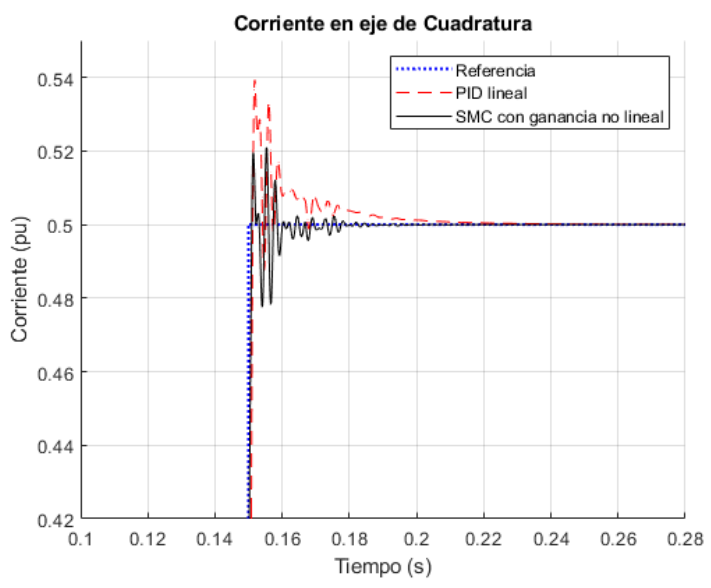

Figura 7: Acercamiento a la respuesta de corriente en eje de cuadratura ante un pulso en la entrada.

En la tabla 3 se calculó el error acumulado integral para el PID lineal

De acuerdo con la estructura del sistema electrónico implementado, el encargado de compensar variaciones en la magnitud de voltaje es el bus DC del sistema, dado que el inversor solo regula la corriente para el suministro de energía a la red.

En la figura 9 se puede apreciar la respuesta del sistema ante la perturbación y en la Figura 10 se puede apreciar un acercamiento a la respuesta del control PID no lineal. Fue necesario realizar un acercamiento dado que el sistema controlador por el PID no lineal presenta una afectación mucho más pequeña que en el caso del PID lineal.

Tabla 3: Desempeño del controlador PID no lineal y del controlador PID lineal.
Control Implementado

PID lineal

PID no lineal

Mejora obtenida
IAE

916.02

$97.12 \%$
Gracias a la perturbación se puede apreciar que la ganancia no lineal implementada en los controladores hace que las acciones de control sean muy fuertes cuando el error del sistema es grande. Esto hace que el sistema alcance las referencias y se recupere de perturbaciones mucho más rápido que cuando se tienen ganancias fijas. Así mismo, se ve que las oscilaciones se reducen mucho debido a que, una vez que el error disminuye, la acción de control también decrece. 


\section{Unach}

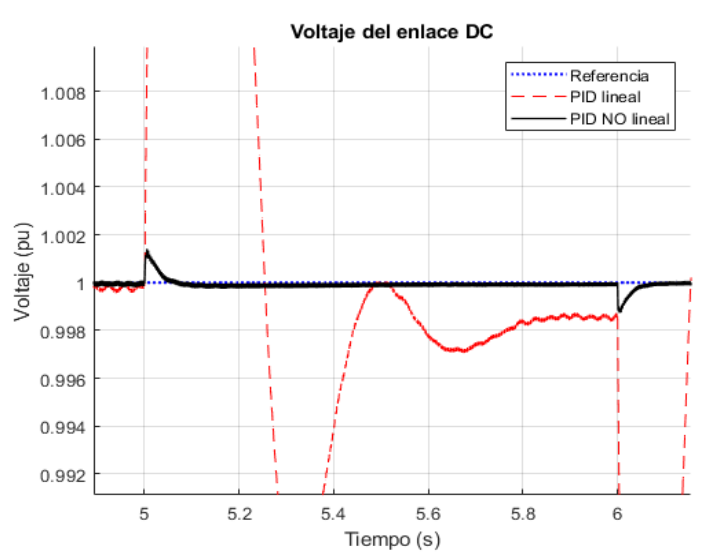

Figura 10: Acercamiento a la respuesta del sistema ante una perturbación.

\section{Conclusiones}

En el presente estudio se proponen dos estrategias de control no lineal, uno con una estructura PID y otra con SMC, para regular el suministro de corriente y estabilización de voltaje en aerogeneradores conectados a un sistema electrónico de potencia. En las estrategias de control se implementó una ganancia no lineal que se calcula en función del error del sistema. Los resultados se compararon con controladores lineales clásicos de tipo PID.

El controlador PID no lineal implementado para el control de voltaje y el SMC implementado para el control de corriente presentaron un mejor desempeño que los controles PID lineales con los que se los comparó, donde en el voltaje DC se obtuvieron mejoras en el orden de $97 \%$ y en la corriente del inversor del $5 \%$ y $33 \%$.

La ganancia no lineal implementada en las dos propuestas, a pesar de tener una estructura simple, presenta notables beneficios en el desempeño de los controladores, sobre todo ante cambios fuertes de referencia y ante perturbaciones externas. Esto sucede debido a que la magnitud de la constante no lineal es grande cuando el error es elevado y es pequeña cuando el sistema se encuentra próximo a la referencia. Como resultado, el sistema se estabiliza rápido, pero presenta menos oscilaciones que un sistema con ganancias constantes.

El sistema fue sometido a una perturbación fuerte en forma de caída de voltaje en los terminales del sistema eléctrico, donde se pudo comprobar que las propuestas no lineales se recuperan más rápido y con menos oscilaciones que los controladores lineales.

\section{Conflicto de Interés}

Los autores informan que no existe conflicto de interés de alguna naturaleza.

\section{Agradecimiento}

Pablo Proaño agradece al programa de becas de la Escuela Politécnica Nacional y al Departamento de Energía Eléctrica por el auspicio para la realización de este trabajo.

\section{Referencias}

Abbas, F. A., Abdulsada, M. A., \& Abusief, F. R. (2011). Speed Control of Wind Turbine by Using PID Controller. Eng. \& Tech. Journal, 29(1), 7. https://doi.org/10.13140/2.1.3717.5680

Bianconi, E., Calvente, J., Giral, R., Mamarelis, E., Petrone, G., Ramos-Paja, C. A., Vitelli, M. (2013). A fast current-based MPPT technique employing sliding mode control. IEEE Transactions on Industrial Electronics, 60(3), 1168-1178. https://doi.org/10.1109/TIE.2012.2190253

Boukhezzar, B., \& Siguerdidjane, H. (2010). Comparison between linear and non-linear control strategies for variable speed wind turbines. Control Engineering Practice, 18(12), 1357-1368.

https://doi.org/10.1016/j.conengprac.2010.06.010

Camacho, O., \& Rojas, R. (2002). A General Sliding Mode Controller for Nonlinear Chemical Processes. Journal of Dynamic Systems, Measurement, and Control, 122(4), 650. https://doi.org/10.1115/1.1318351

Camacho, O., \& Smith, C. A. (2000). Sliding mode control: An approach to regulate non-linear chemical processes. ISA Transactions, 39(2), 205-218. https://doi.org/10.1016/s0019-0578(99)00043-9

Capito, L., Proaño, P., Camacho, O., Rosales, A., \& Scaglia, G. (2016). Experimental comparison of control strategies for trajectory tracking for mobile robots. International Journal of Automation and Control, 10(3), 308.

https://doi.org/10.1504/ijaac.2016.077591

Fernández, J. I. (2011). Controladores PI con acción de reset (Proyecto Final de Carrera, Universitat Autonoma de Barcelona). Recuperado de https://sistemamid.com/panel/uploads/biblioteca/201 7-08-13_12-02-41141863.pdf

Lei, Z., \& Zhou, Y. (2018). A kind of non-linear PID controller for Refrigeration Systems based on Vapour Compression. IFAC-PapersOnLine, 51 (4), 716-721. https://doi.org/10.1016/j.ifacol.2018.06.188

Liu, Y., Wang, Z., Xiong, L., Wang, J., Jiang, X., Bai, G., Liu, S. (2018). DFIG wind turbine sliding mode control with exponential reaching law under variable wind speed. International Journal of Electrical Power and Energy Systems, 96 (October 2017), 253260. https://doi.org/10.1016/j.ijepes.2017.10.018

Pöschke, F., Fortmann, J., \& Schulte, H. (2017). Non-linear wind turbine controller for variable power generation in full load region. In Proceedings of the American Control Conference (pp. 1395-1400). Seattle, WA, 


\section{Unach}

USA: IEEE

https://doi.org/10.23919/ACC.2017.7963148

Qi, Y., \& Meng, Q. (2012). The application of fuzzy PID control in pitch wind turbine. Energy Procedia, 16(PART C), 1635-1641.

https://doi.org/10.1016/j.egypro.2012.01.254

Rodríguez-Mariano, A., Reynoso-Meza, G., PáramoCalderón, D. E., Chávez-Conde, E., García-Alvarado, M. A., \& Carrillo-Ahumada, J. (2015). Análisis del desempeño de controladores lineales sintonizados en diferentes estados estacionarios del biorreactor de Cholette mediante tecnicas dedecision multi-criterio. Revista Mexicana de Ingeniera Quimica, 14(1), 167204.

Su, Y. X., Sun, D., \& Duan, B. Y. (2005). Design of an enhanced non-linear PID controller. Mechatronics, 15(8), 1005-1024.

https://doi.org/10.1016/j.mechatronics.2005.03.003 